\title{
In-plane substitution effect on the magnetic properties of two-dimensional Spin-gap system $\mathrm{SrCu}_{2}\left(\mathrm{BO}_{3}\right)_{2}$
}

\author{
G. T. liu, ${ }^{1}$ J. L. Luo, ${ }^{1, \text { ศ }}$ Y. Q. Guo, ${ }^{1}$ S. K. Su, ${ }^{1}$ P. Zheng, ${ }^{1}$ N. L. Wang, ${ }^{1}$ D. Jin,,${ }^{1}$ and T. Xiang ${ }^{2}$ \\ ${ }^{1}$ Beijing National Laboratory of Condensed Matter Physics, Institute of Physics, \\ Chinese Academy of Sciences, Beijing 100080, People's Republic of China \\ ${ }^{2}$ Institute of Theoretical Physics and Interdisciplinary Center of Theoretical Studies, \\ Chinese Academy of Science, P.O. Box 2735, Beijing 100080, People's Republic of China
}

(Dated: June 24, 2018)

\begin{abstract}
A series of in-plane substituted compounds, including $\mathrm{Cu}$-site $\left(\mathrm{SrZn}_{x} \mathrm{Cu}_{2-x}\left(\mathrm{BO}_{3}\right)_{2}\right)$, and B-site $\left(\mathrm{SrCu}_{2}\left(\mathrm{Si}_{x} \mathrm{~B}_{1-x} \mathrm{O}_{3}\right)_{2}\right)$ substitution, were synthesized by solid state reaction. X-ray diffraction measurements reveal that these compounds are single-phase materials and their in-plane lattice parameter depends systematically on the substituting content $x$. The magnetic susceptibility in different magnetic fields, the magnetization at different temperatures, and the resistivity at room temperature were measured, respectively. It is found that the spin gap deduced from the magnetic susceptibility measurements decreases with increasing of $x$ in both $\mathrm{Cu}$ - and B-site substitution. No superconductivity was found in these substituted compounds.

PACS numbers: 75.40.Cx, 75.10.Jm, 75.50.Ee
\end{abstract}

The psedudogap observed in the high- $T_{c}$ cuprates has stimulated intensified interest on the investigations of systems with spin-gap. A novel spin-gap system discovered recently is strontium copper borate $\mathrm{SrCu}_{2}\left(\mathrm{BO}_{3}\right)_{2}[1]$. This material is a realization of the two-dimensional spin$1 / 2$ Shastry-Sutherland model[2]. It has a tetragonal structure constructed with alternating $\mathrm{CuBO}_{3}$ and $\mathrm{Sr}$ planes [3]. At room temperature, the lattice parameters are $a=8.995 \AA$ and $c=6.649 \AA$. In $\mathrm{CuBO}_{3}$ plane, all $\mathrm{Cu}^{2+}$ ions with spin- $1 / 2$ are located at crystallographically equivalent sites, and the nearest two $\mathrm{Cu}^{2+}$ ions form a dimer unit. The dimer units are connected orthogonally through $\mathrm{BO}_{3}$ triangles. The magnetic properties of $\mathrm{SrCu}_{2}\left(\mathrm{BO}_{3}\right)_{2}$ can be effectively described by the two-dimensional Heisnberg model:

$$
H=J \sum_{N N} S_{i} S_{j}+J^{\prime} \sum_{N N N} S_{i} S_{j}
$$

where $J$ and $J^{\prime}$ are the intradimer and interdimer exchange constants. The phase diagram of this ShastySutherland-type model has been studied [4, 5, 6, 7]. The ground state of the model is gapped and described approximately by a product of local dimer singlets when $J^{\prime} / J<0.68$ 2, 8]. Recently experimental results for magnetic susceptibility [1, 9], Cu nuclear quadrupole resonance 1], high-field magnetization [1], electron-spin resonance 10, Raman scattering 11, inelastic neutron scattering 12, 13], and specific heat 14] showed that the spin gap of this material is $\sim 30 \mathrm{~K}$. This material shows a plateaus in the magnetization curve 1, 15. In order to explain this phenomenon, great effort has been devoted to the study of excitation state. It was found that the magnetization plateau is associated with a superstructure induced by localized spin excitations [8, [16]. For

*Electronic address: JLLuo@aphy.iphy.ac.cn
$\mathrm{SrCu}_{2}\left(\mathrm{BO}_{3}\right)_{2}$, the ratio $J^{\prime} / J$ is close to its critical value. The value of $J^{\prime} / J$ can be changed by applying pressure or substitution [17, 18]. On doping, this system may select other ordered (or disordered) states so that electrons or holes can move freely 17.

Recently it was suggested theoretically that this material can become superconducting upon electron or hole doping 7, 18, 19, 20]. A number of different superconducting states were suggested at different doping levels [7, 19, 20]. To examine these theoretical proposals, Kudo 21], Lappas 22], Choi 23] and Zorko 24, 25] et al investigated doping effect of non-magnetic ions on $\mathrm{SrCu}_{2}\left(\mathrm{BO}_{3}\right)_{2}$. They found that the spin gap is hardly to be suppressed by small substitution of non-magnetic impurities. Our previous work [26] indicates that substituting $\mathrm{Sr}$ can significantly reduce the spin gap and the electrical resistivity, especially for La-doping. But the high resistivity, in the order of $\sim 10^{8} \Omega \mathrm{m}$, indicating that they are all insulators. These results suggested that these doped carriers are all almost localized. The possible reason comes from the $\mathrm{B}-\mathrm{O}$ bond, which adopts $s p^{2}$ hybridization. The bond is so stable that these doped carriers are localized in the $\mathrm{CuBO}_{3}$ plane. In this paper, we expect the in-plane substitution might destroy or distort $s p^{2}$ hybridization and form some conducting channels in $\mathrm{CuBO}_{3}$, thus superconductivity maybe occurs. Unfortunately, we found no such an effect. However, we find that both Zn- and Si-substituting has pronounced effects on the dimer structure and magnetic properties.

The samples of $\mathrm{SrZn}_{x} \mathrm{Cu}_{2-x}\left(\mathrm{BO}_{3}\right)_{2}$ (Cu-site substitution) and $\mathrm{SrCu}_{2}\left(\mathrm{Si}_{x} \mathrm{~B}_{1-x} \mathrm{O}_{3}\right)_{2}$ (B-site substitution) with different substituting content $x$ were prepared by heating stoichiometric mixtures of $\mathrm{SrCO}_{3}, \mathrm{CuO}, \mathrm{B}_{2} \mathrm{O}_{3}, \mathrm{ZnO}$ and $\mathrm{SiO}_{2}$ with high purity. Initial chemicals were mixed carefully, followed by heat treatment between 820 to $860^{\circ} \mathrm{C}$ in air for one week with several intermediate grindings.

Powder x-ray diffraction (XRD) measurements at room temperature were done to examine the phase 
TABLE I: Lattice parameters, $\chi_{0}\left(\times 10^{-3} \mathrm{emu} /(\mathrm{mol} \mathrm{Cu})\right), C^{\prime}\left(\times 10^{-3} \mathrm{emu} \mathrm{K} /(\mathrm{mol} \mathrm{Cu})\right), \Delta$, resistivity $\rho\left(\times 10^{8} \Omega \mathrm{m}\right)$, effective moments $\mu_{\text {eff }}$, and the atomic ratio in $\operatorname{SrZn}_{x} \mathrm{Cu}_{2-x}\left(\mathrm{BO}_{3}\right)_{2}$ and $\mathrm{SrCu}_{2}\left(\mathrm{Si}_{x} \mathrm{~B}_{1-x} \mathrm{O}_{3}\right)_{2}$.

\begin{tabular}{|c|c|c|c|c|c|c|c|c|c|c|c|c|c|}
\hline Formula & $x$ & $a(\AA)$ & $c(\AA)$ & $\chi_{0}$ & $C^{\prime}$ & $\Delta(\mathrm{K})$ & $\rho$ & $\mu_{\text {eff }}\left(\mu_{B}\right)$ & $\mathrm{Cu}$ & B & $\mathrm{Sr}$ & $\mathrm{Zn}$ & $\mathrm{Si}$ \\
\hline \multirow{4}{*}{$\mathrm{SrZn}_{x} \mathrm{Cu}_{2-x}\left(\mathrm{BO}_{3}\right)_{2}$} & 0 & 8.995 & 6.649 & -0.740 & 10.57 & 21.5 & 4.158 & 0.110 & & & & & \\
\hline & 0.05 & 8.990 & 6.648 & -0.898 & 12.59 & 20.1 & 1.045 & 0.156 & 1.95 & 1.97 & 1.00 & 0.05 & \\
\hline & 0.10 & 8.989 & 6.649 & -1.043 & 14.02 & 16.7 & 0.957 & 0.193 & 1.90 & 1.93 & 1.00 & 0.09 & \\
\hline & 0.15 & 8.984 & 6.647 & -2.889 & 32.77 & 15.0 & 0.898 & 0.212 & 1.79 & 1.95 & 1.00 & 0.11 & \\
\hline \multirow{4}{*}{$\mathrm{SrCu}_{2}\left(\mathrm{Si}_{x} \mathrm{~B}_{1-x} \mathrm{O}_{3}\right)_{2}$} & 0.04 & 8.990 & 6.649 & -0.828 & 13.41 & 20.2 & 3.450 & 0.177 & & & & & \\
\hline & 0.06 & 8.989 & 6.648 & -1.291 & 17.94 & 17.9 & 3.238 & 0.226 & 1.95 & 1.77 & 1.00 & & 0.15 \\
\hline & 0.08 & 8.987 & 6.649 & -1.796 & 26.36 & 16.4 & 2.106 & 0.280 & 1.94 & 1.75 & 1.00 & & 0.20 \\
\hline & 0.10 & 8.986 & 6.649 & -2.897 & 33.21 & 15.1 & 1.763 & 0.284 & 2.02 & 1.72 & 1.00 & & 0.22 \\
\hline
\end{tabular}

purity. Figure 1 shows the typical XRD patterns of $\mathrm{SrZn}_{x} \mathrm{Cu}_{2-x}\left(\mathrm{BO}_{3}\right)_{2}$ and $\mathrm{SrCu}_{2}\left(\mathrm{Si}_{x} \mathrm{~B}_{1-x} \mathrm{O}_{3}\right)_{2}$. The diffraction peaks can be well indexed on the known tetragonal unit cell with space group of $I \overline{4} 2 \mathrm{~m}$. It indicates that these samples are single phase in the range of $0 \leq x \leq 0.15$ for $\operatorname{SrZn}_{x} \mathrm{Cu}_{2-x}\left(\mathrm{BO}_{3}\right)_{2}$ and $0 \leq x \leq 0.1$ for $\mathrm{SrCu}_{2}\left(\mathrm{Si}_{x} \mathrm{~B}_{1-x} \mathrm{O}_{3}\right)_{2}$. However, no pure samples can be obtained if substitution content $x$ is further increased. Table 1 shows the structure parameters obtained from the least-squares calculation of $2 \theta$ values. Figure 2 shows the relative change of lattice parameters as a function of $x$. It can be seen that the in-plane parameter $a$ decreases monotonously with increasing $x$. However, the $c$-axis parameter $c$ remains almost unchanged. This is different from the out-of-plane substitution where $a$ keeps almost unchanged but $c$ changes monotonously with doping 26]. The induction-coupled plasma (ICP) method was employed to determine the atomic ratio. The experimental

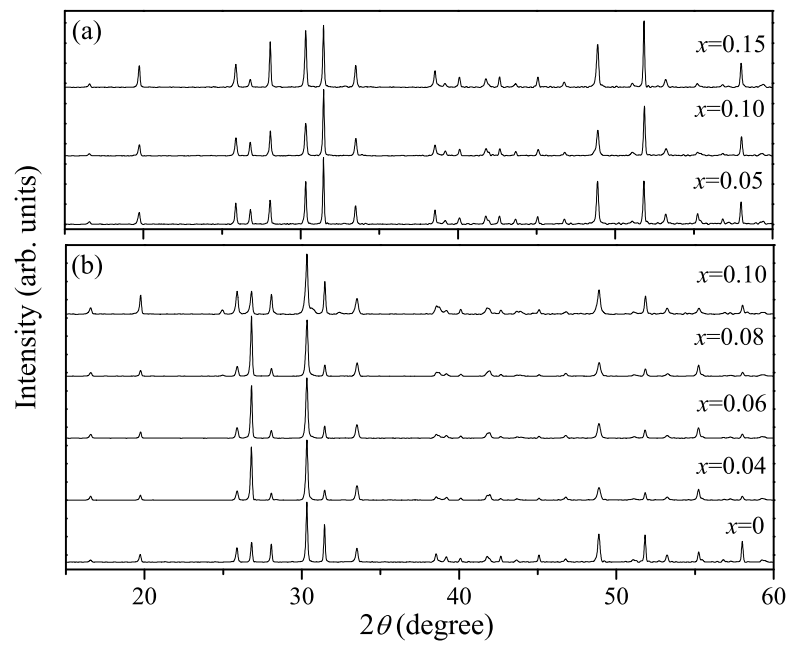

FIG. 1: The x-ray powder diffraction patterns of (a) $\mathrm{SrZn}_{x} \mathrm{Cu}_{2-x}\left(\mathrm{BO}_{3}\right)_{2}$ with $x \leq 0.15$, and (b) $\mathrm{SrCu}_{2}\left(\mathrm{Si}_{x} \mathrm{~B}_{1-x} \mathrm{O}_{3}\right)_{2}$ samples with $x \leq 0.1$.

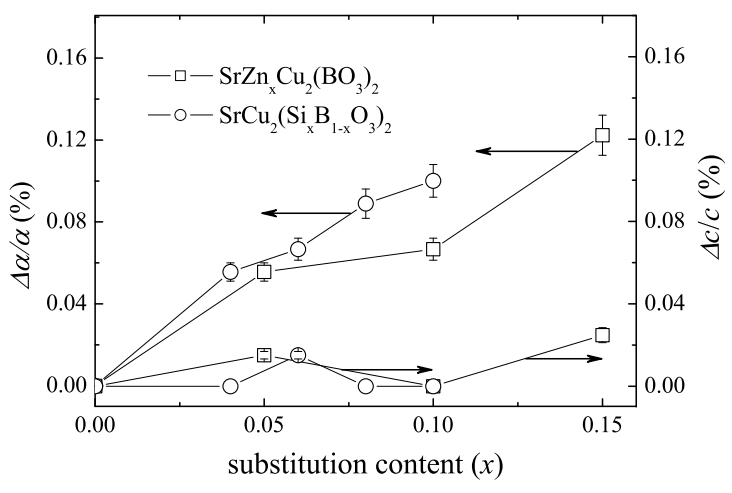

FIG. 2: Substituting content dependence of lattice parameters for $\mathrm{SrZn}_{x} \mathrm{Cu}_{2-x}\left(\mathrm{BO}_{3}\right)_{2}$ and $\mathrm{SrCu}_{2}\left(\mathrm{Si}_{x} \mathrm{~B}_{1-x} \mathrm{O}_{3}\right)_{2}$.

results listed in Table 1 are close to the nominal composition. These results suggest that the substitution takes place in the $\mathrm{CuBO}_{3}$ plane.

The magnetization $M$ was measured using a commercial Quantum Design PPMS. The resistivity at room temperature was measured with a four-probe method using the Keithley low current source model 6487 Picoammeter.

Figure 3 shows the dc magnetic susceptibility $\chi=M / H$ as a function of temperature $T$ for $\operatorname{SrZn}_{x} \mathrm{Cu}_{2-x}\left(\mathrm{BO}_{3}\right)_{2}$ with $x=0.05,0.10$, and 0.15 at $1.0 \mathrm{~T}$ from 2 to $20 \mathrm{~K}$. For all Zn-substituting samples, $\chi$ follows the CurieWeiss law in high temperature regime $(T>20 \mathrm{~K})$. At $\sim 10 \mathrm{~K}$ (defined as $T_{S G}$ ), a spin gap opens and $\chi$ drops sharply. The susceptibility behavior is typical for a lowdimensional spin-gap system, and it is similar to our previous out-of-plane doping result 26]. A Curie-like upturn contributed by magnetic impurities [1, 24] is observed below $4 \mathrm{~K}$. Compared with our previous Y-doping case [26], the magnitude of upturn here is more pronounced. The reason is some $\mathrm{Cu}^{2+}$ were replaced by spinless $\mathrm{Zn}^{2+}$, which creates some individual $\mathrm{Cu}^{2+}$ with spin- $1 / 2$ by destroying a certain number of $\mathrm{Cu}^{2+}-\mathrm{Cu}^{2+}$ dimers. As 


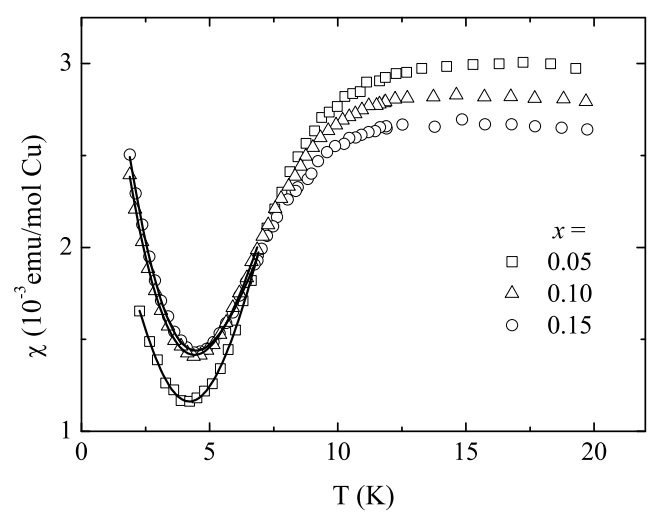

FIG. 3: Temperature dependence of the magnetic susceptibility for $\mathrm{SrZn}_{x} \mathrm{Cu}_{2-x}\left(\mathrm{BO}_{3}\right)_{2}$ in a field of $1.0 \mathrm{~T}$, the solid lines are the fitting results with Eq. (2).

it is known that the contribution of magnetic impurities to magnetic susceptibility follows the Curie-Weiss law, $\chi \sim 1 /(T-\theta)$. However, the magnetic susceptibility of anti-ferromagnetic dimers satisfies exponential law, $\chi \sim e^{-1 / T}$. Therefore, the contribution of these magnetic impurities is very notable in low temperature range. The upturn of $\chi$ at low temperatures rises with increasing $x$, which indicates that more magnetic impurities were introduced. These magnetic-impurity concentration can be reflected by effective moment $\mu_{\text {eff }}$. From the temperature dependence of $\chi$ at low temperatures, we can determine $\mu_{\text {eff }}\left(C^{\prime}=N \mu_{\text {eff }}^{2} / 3 k_{B}\right)$ and the excitation gap $\Delta$ using the following relation [26]:

$$
\chi=\frac{C^{\prime}}{T-\theta^{\prime}}+a \exp (-\Delta / T)+\chi_{0} .
$$

The first term represents the contribution of spin- $1 / 2$ magnetic impurities. The second term represents the contribution from the anti-ferromagnetic dimer structure. The third term is a small constant which includes

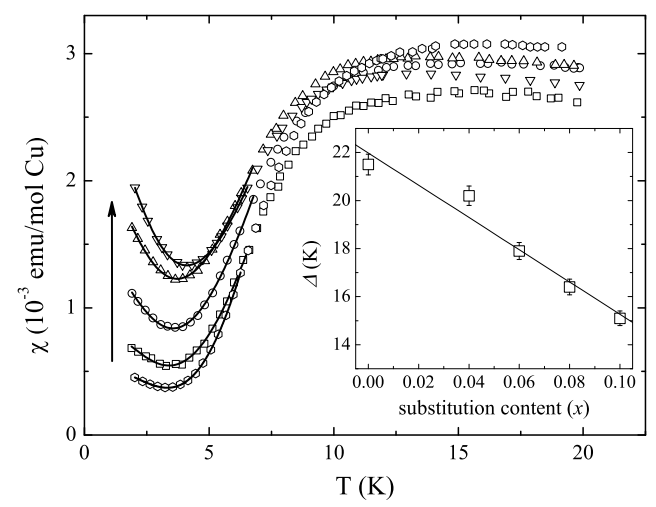

FIG. 4: Temperature dependence of the magnetic susceptibility for $\mathrm{SrCu}_{2}\left(\mathrm{Si}_{x} \mathrm{~B}_{1-x} \mathrm{O}_{3}\right)_{2}$ in a field of $1.0 \mathrm{~T}$, the solid lines are the fitting results with Eq. (2), the arrow direction denotes the increase of $x$ from 0 to 0.10 . The inset shows the spin gap $\Delta$ at different substituting content $x$. the diamagnetic contribution of both the sample holder and the sample itself. The fitting results of $\chi_{0}, C^{\prime}, \Delta$ as well as $\mu_{\text {eff }}$ are given in Table 1 . It can be seen that, with $x$ increased from 0 to $0.15, \Delta$ is suppressed from 21.5 to $15 \mathrm{~K}$, while $\mu_{\text {eff }}$ increased from 0.110 to $0.212 \mu_{\mathbf{B}}$.

Figure 4 shows a change of $\chi$ for $\mathrm{SrCu}_{2}\left(\mathrm{Si}_{x} \mathrm{~B}_{1-x} \mathrm{O}_{3}\right)_{2}$ plotted as a function of $T$. We find $\chi$ has an analogous behavior as that of Zn-substituting samples discussed above. With the same method as treating $\mathrm{Zn}$ substituting samples, $\Delta$ and $\mu_{\text {eff }}$ can be obtained for Si-substituting samples, which are listed in Table 1. From the inset of Fig. 4, one can see that $\Delta$ reduces monotonously with $x$ increased. From the value of the spin gap listed in Table 1, one can see that Zn- and Si-substituting have a similar effect on the reduction of the spin gap. It should be noted that the spin gap deduced from the magnetic susceptibility data is $\sim 10 \mathrm{~K}$ less than that from ESR [10], Raman scattering [1], neutron scattering [12, 13], specific heat 14], and NMR 16] measurements. The reason maybe comes from the upturn of susceptibility. However, it does not affect the qualitative analysis of the effect of substitution on the spin gap.

In order to further investigate the intrinsic nature of magnetic state of in-plane substituted compound. We have measured $\chi(T)$ at different magnetic fields $H$ and $M(H)$ at different temperatures $T$ for $\mathrm{SrCu}_{2}\left(\mathrm{Si}_{0.1} \mathrm{~B}_{0.9} \mathrm{O}_{3}\right)_{2}$. Figure 5 shows $\chi$ as a function of $T$ up to $12 \mathrm{~T}$. The low-temperature susceptibility upturn is gradually suppressed by rasing external fields. From the inset of Fig. 5, a linear field dependence of spin gap is observed, which could be explained by Zeeman splitting. This result is also consistent with that derived from specific heat data for out-of-plane doping cases 26]. Figure 6 shows the $M(H)$ at different temperatures. At low temperatures $\left(T<T_{S G}\right)$, the initial linear field dependence of $M(H)$ signifying that $M(H)$ is dominated by magnetic impurity; with $H$ further increased, $M(H)$ gradually increases with $H$, in this stage external field $H$ firstly saturates the $M(H)$ of magnetic impurity and then breaks some $\mathrm{Cu}^{2+}-\mathrm{Cu}^{2+}$ dimers. At higher temperatures $\left(T \geq T_{S G}\right)$, the linear relationship between

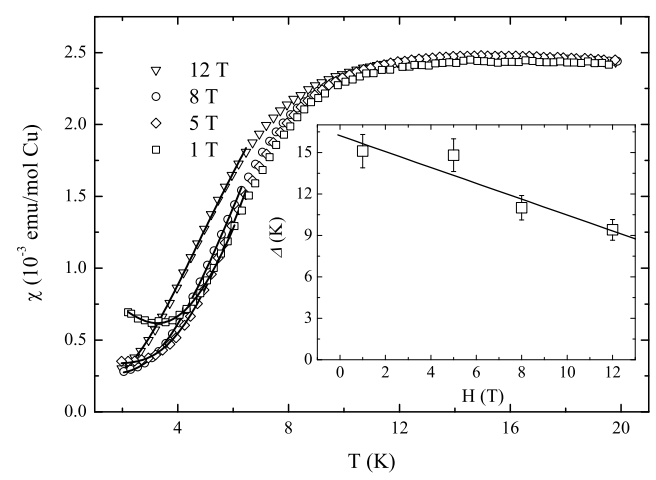

FIG. 5: Magnetic susceptibility $\chi$ as a function of temperature $T$ of $\mathrm{SrCu}_{2}\left(\mathrm{Si}_{0.1} \mathrm{~B}_{0.9} \mathrm{O}_{3}\right)_{2}$ up to $12 \mathrm{~T}$. The inset shows the spin gap $\Delta$ as a function of fields $H$. 


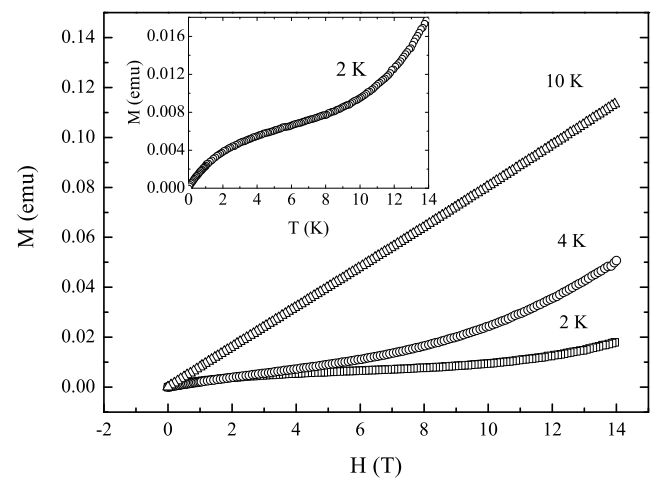

FIG. 6: Magnetization $M$ as a function of magnetic fields $H$ of $\mathrm{SrCu}_{2}\left(\mathrm{Si}_{0.1} \mathrm{~B}_{0.9} \mathrm{O}_{3}\right)_{2}$. The inset shows the magnetization at $2 \mathrm{~K}$.

$M(H)$ and $H$ can be interpreted as $\mathrm{Cu}^{2+}-\mathrm{Cu}^{2+}$ dimers were broken by thermal fluctuation. We measured $\chi$ with zero-field-cooled (ZFC) as well as field-cooled (FC), no spin-frozen or hysteresis was found. These results suggest that there are two kinds of magnetic systems in $\mathrm{SrCu}_{2}\left(\mathrm{Si}_{0.1} \mathrm{~B}_{0.9} \mathrm{O}_{3}\right)_{2}$. One is dimer system, and the other one is magnetic impurity system induced by substitution.

The resistivity $\rho$ measurement results are listed in Table 1 . The resistivity decreases with increasing of substituting content for both $\mathrm{Cu}$ - and B-site substitutions.
However, we find that the decrease in resistivity for inplane substitution is much less than that for out-of-plane substitution 26], for example, the resistivity for $10 \% \mathrm{La}$ doping is about 2 orders less than that for undoped $\mathrm{SrCu}_{2}\left(\mathrm{BO}_{3}\right)_{2}$. In case of $\mathrm{Zn}$-substituting, no carriers are introduced since $\mathrm{Zn}^{2+}$ ion has the same valence to that of $\mathrm{Cu}^{2+}$. While for Si-substituting case, $s p^{2}$ hybridization of $\mathrm{B}-\mathrm{O}$ bond might not be destroyed, therefore no additional conducting channels were formed. The balance of electrovalence between $\mathrm{Si}^{4+}$ and $\mathrm{B}^{3+}$ may be adjusted by oxygen content.

In conclusion, we have synthesized a series of in-plane substituted materials, including $\mathrm{Cu}-$ site $\left(\mathrm{SrZn}_{x} \mathrm{Cu}_{2-x}\left(\mathrm{BO}_{3}\right)_{2}\right)$ and B-site substitution $\left(\mathrm{SrCu}_{2}\left(\mathrm{Si}_{x} \mathrm{~B}_{1-x} \mathrm{O}_{3}\right)_{2}\right)$. Their structures and magnetic properties have been systematically studied. XRD analysis suggests that these compounds are single-phase materials and their in-plane lattice parameter depends systematically on the substituting content $x$. The magnetism measurements indicate that there are two kinds of magnetic systems in the substituted samples. One is magnetic impurity system, and the other one is dimer structure system. The spin gap can be reduced by element substitution. No superconductivity appeared in the in-plane substituted samples.

This work is supported by the National Natural Science Foundation of China.
[1] H. Kageyama, K. Yoshimura, R. Stern, N. V. Mushnikov, K. Onizuka, M. Kato, K. Kosuge, C. P. Slichter, T. Goto, and Y. Ueda, Phys. Rev. Lett. 82, 3168 (1999).

[2] B. S. Shastry and B. Sutherland, Physica B 108, 1069 (1981).

[3] R. W. Smith and D. A. Keszler, J. Solid. State. Chem. 93, 430 (1991).

[4] A. Koga, and N. Kawakami, Phys. Rev. Lett. 84, 4461 (2000).

[5] W. Zheng, J. Oitmaa and C. Hamer, Phys. Rev. B 65, 014408 (2002).

[6] D. Carpentier, and L. Balents, Phys. Rev. B 65, 024427 (2002).

[7] C. H. Chung, and Y. B. Kim, Phys. Rev. Lett. 93, 207004 (2004).

[8] S. Miyahara and K. Ueda, Phys. Rev. Lett. 82, 3701 (1999).

[9] H. Kageyama, K. Onizuka, T. Yamauchi, Y. Ueda, S. Hane, H. Mitamura, T. Goto, K. Yoshimura, and K. Kosuge, J. Phys. Soc. Jpn. 68, 1821 (1999).

[10] H. Nojiri, H. Kageyama, K. Onizuka, Y. Ueda, and M. Motokawa, J. Phys. Soc. Jpn. 68, 2906 (1999)

[11] P. Lemmens, M. Grove, M. Nishi, G. Güntherodt, V. N. Kotov, H. Kageyama, K. Onizuka, and Y. Ueda, Phys. Rev. Lett. 85, 2605 (2000).

[12] H. Kageyama, M. Nishi, N. Aso, K. Onizuka, T. Yosihama, K. Nukui, K. Kodama, K. Kakurai, and Y. Ueda, Phys. Rev. Lett. 84, 5876 (2000).

[13] O. Cépas, K. Kakurai, L. P. Regnault, T. Ziman, J. P.
Boucher, N. Aso, M. Nishi, H. Kageyama, and Y. Ueda Phys. Rev. Lett. 87, 167205 (2001).

[14] H. Kageyama, H. Suzuki, M. Nohara, K. Onizuka, H. Takagi, and Y. Ueda, Physica B 281, 667 (2000).

[15] K. Onizuka, and J. Phys. Soc. Jpn. 69, 1016 (2000)

[16] K. Kodama, M. Takigawa, M. Horvatic, C. Berthier, H. Kageyama, Y. Ueda, S. Miyahara, F. Becca, and F. Mila, Science. 298, 395 (2002).

[17] C. H. Chung, J. B. Marston and S. Sachdev, Phys. Rev. B 64, 134407 (2001).

[18] P. W. Leung, and Y. F. Cheng, Phys. Rev. B 69, 180403(R) (2004).

[19] B. S. Shastry and B. Kumar, Prog. Theor. Phys. Suppl. 145, 1 (2002).

[20] T. Kimura, K. Kuroki, R. Arita, and H. Aoki, Phys. Rev. B 69, 054501 (2004).

[21] K. Kudo, T. Noji, Y. Koike, T. Nishizaki, and N. Kobayashi, Physica B 329-333, 910-911 (2003).

[22] A. Lappas, A. Schenck, and K. Prassides, Physica B 326, 431-435 (2003).

[23] K. -Y. Choi, Yu. G. Pashkevich, K. V. Lamonova, H. Kageyama, Y. Ueda, and P. Lemmens, Phys. Rev. B 68, 104418 (2003).

[24] A. Zorko and D. Arčon, Phys. Rev. B 65, 024417 (2001).

[25] A. Zorkoa, D. Arčon, C. J. Nuttallb, A. Lappas, Physica B 272-276, e699 (2004).

[26] G. T. Liu, J. L. Luo, T. Xiang, N. L. Wang, Z. H. Wu, X. N. Jing and D. Jin, Phys. Rev. B 71, 014441 (2005). 